\title{
DIFFUSION LIMITED IMMUNOCHEMICAL SENSING
}

\author{
- Bernard J. Geurts \\ Center for Theoretical Physics, \\ Twente University, \\ P.O. Box 217, \\ Enschede $7500 \mathrm{AE}$, \\ The Netherlands
}

\begin{abstract}
The time-dependent surface coverage of antigen-antibody complexes for a sensor in which antigens are bound to surface immobilized antibodies is determined analytically. Assuming a reversible first order reaction between the antigens and antibodies, a model is derived describing the dynamical response of the sensor. The surface coverage is related explicitly to the antigen concentration which is of special interest in experimental situations. The stationary state and short time behaviour are determined explicitly. Several illustrations of the full solution are provided.
\end{abstract}

1. Introduction. The problem of direct immunochemical sensing has received some theoretical attention in recent years (Eddowes, 1987; Stenberg et al., 1986). It is closely related to chemoreception by living cells (Wiegel, 1983; Geurts and Wiegel, 1987). In this paper, a sensor which consists of a fluid layer with antigens at some concentration, on top of an antibody coated surface, is considered. These antigens diffuse through the fluid and can reversibly form complexes with the antibodies at the "sensor surface". The time-dependence of the surface coverage of antibody-antigen complexes is determined. This quantity is of special interest for direct immunochemical sensing, which can be realized in various ways [e.g. optical devices such as plasmonsensors (Eddowes, 1987)]. This time-dependent problem is treated using the boundary condition approach of DeLisi and Wiegel (1981). In an experimental situation one is interested in determining the (unknown) antigen concentration of a sample and/or in finding reaction and sensor parameters. These quantities are explicitly related to the measurable surface coverage, and immunochemical sensing regarded as the formation of antibody-antigen complexes.

Immunochemical sensing devices enjoy a considerable amount of experimental interest presently. The formation of antibody-antigen complexes at the "sensor surface" changes physical parameters of that surface (e.g. the diffraction index) which can be measured in order to obtain the concentration of antigen in a sample. In this paper antigens which can either be freely diffusing in the medium or, when bound, are bound to exactly one recepting site on an antibody are considered [this antigen can be any small hapten such as 
progesteron (Stryer, 1981)]. As an example of the antibodies used, one may think of an IgG-molecule (immunoglobulin). This is a Y-shaped molecule with two receptor sites; hence IgG is bivalent, it can bind at most two antigens simultaneously. For a discussion of IgG and the basic geometry see Stollar (1981) and Wiegel et al. (1987). The antigens will be referred to as ligands and the receptor sites as receptors in this paper. So, in the case of IgG as antibody there are twice as many receptors as IgG molecules. One could extend the model to include multivalent ligands as well by including the multivalent character into the reaction kinetics describing the ligand-receptor reaction at the surface, this as has been done by Stenberg et al. (1986) for the case of bivalent ligands. It implies the inclusion of extra reaction parameters describing the various different ways and transitions between them, in which a ligand can be bound. This extension is not considered here, only monovalent ligands which can bind to recepting sites on (multivalent) antibodies are discussed.

Consider $N_{0}$ receptors distributed uniformly over a surface at $x=0$. These receptors can reversibly form complexes with the ligands in the outside medium. The transport of the ligands is governed by diffusion. Due to the reversible character of the receptor-ligand reaction at the receptor coated surface, complexes are continuously being created and annihilated. If a complex disintegrates not only does a receptor become free for further ligand binding but a ligand is also reinserted into the medium close to the surface and free to diffuse. This effect is taken into account by the incorporation of a surface source term into the diffusion equation governing the ligand distribution. The strength of this source term is proportional to the number of receptor-ligand complexes.

In Section 2 the model describing the evolution of the surface coverage is formulated. The stationary state is considered in Section 3. The full analytical solution is derived in Section 4 and the short time behaviour is determined explicitly. In the analytical solution, the ligand distribution and surface coverage are expressed in terms of the ligand distribution at the sensor surface. This implies a set of simultaneous non-linear integral equations for the distribution at the sensor surface and the surface coverage. The numerical approach to this system of integral equations is described in Section 5. The reader not interested in the mathematical treatment of the problem can continue reading in Section 6 in which results obtained for the surface coverage are discussed.

2. Model Describing the Evolution of the Surface Coverage. Consider a receptor coated plane surface of area $A$. Denote the perpendicular distance to this surface by $x$. On this surface $N_{0}$ receptors are distributed uniformly. In the outside medium which extends to $x=l$, ligands are found which can form 
complexes with these receptors. The ligands follow erratic paths and their translational motion is characterized by a diffusion coefficient $D$. Let $c(x, t)$ be the number density of free, that is unbound, ligands at $x$ at time $t$. A mass flow is associated with a gradient in the ligand distribution. Assuming Fickian diffusion, the number of ligands passing through a plane surface at $x$ perpendicular to the $x$-axis per unit of time is given by:

$$
J(x, t)=A D \partial_{x} c(x, t) .
$$

This implies that per unit of time $A D \partial_{x} c(0, t)$ ligands are bound to the surface at $x=0$. Moreover, let $n(t)$ denote the number of receptor-ligand complexes at time $t$. If the reaction between receptors and ligands is assumed to be of first order and reversible then $\gamma n(t)$ complexes will disintegrate per unit of time, where $\gamma$ is the reverse reaction constant. Hence:

$$
\frac{\mathrm{d} n(t)}{\mathrm{d} t}=A D \partial_{x} c(0, t)-\gamma n(t) .
$$

As initial condition we take $n(0)=0$, i.e. a clean surface. Notice that $n(t)$ can be obtained straightforwardly from equation (2) if $\partial_{x} c(0, t)$ is known. We next describe the equation governing the evolution of the ligand distribution $c(x, t)$.

If a receptor-ligand complex disintegrates not only is a receptor freed for further ligand binding but simultaneously a ligand is released into the medium close to $x=0$, thus influencing the ligand distribution. This can be described by introducing a ligand surface source term into the diffusion equation (Carslaw and Jaeger, 1959). Let $\tilde{n}=n / A$, then the ligand distribution is governed by:

$$
\partial_{t} c(x, t)=D \partial_{x x} c(x, t)+\gamma \tilde{n}(t) \delta(x-\varepsilon)
$$

where $0<\varepsilon \ll l$. The parameter $\varepsilon$ indicates that the ligands coming from disintegrating complexes are released at $x=\varepsilon$. It is inserted for mathematical convenience in order to avoid conflict with the boundary condition at $x=0$. Eventually interest resides in the limit at which $\varepsilon \rightarrow 0$. The above equations constitute the basic model. These must be supplemental with appropriate initial and boundary conditions.

Assuming that initially ligands are found at uniform concentration, i.e.

$$
c(x, 0)=c_{0} .
$$

At the outer boundary $(x=l)$ one is free to choose the boundary condition. Throughout this paper two types of sensor will be simultaneously considered. If at $x=l$ a membrane is placed in contact with a large reservoir of ligands in which the ligand concentration is kept equal to $c_{0}$ then:

$$
c(l, t)=c_{0} .
$$


Such sensors will be referred to as type I sensors. If, however, an impermeable surface is placed at $x=l$, then the ligand current at $x=l$ vanishes. Hence, in view of equation (1):

$$
\partial_{x} c(l, t)=0
$$

These are called type II sensors.

The boundary condition at $x=0$ is rather special (DeLisi and Wiegel, 1981). Assuming that the receptors can be characterized by a single linear dimension $s$ (Wiegel, 1983), the receptors can be regarded as being approximated by perfectly absorbing disks of radius $s$. The number of ligands being bound per unit of time, if the receptor surface density is low, can then be shown to be given by:

$$
J(t)=N(t) 4 s D c(a, t),
$$

where $s \ll a \ll l$ and $N(t)$ is the total number of free receptors at time $t$, that is receptors which at time $t$ do not form a complex with a ligand. However, as was argued before $J(t)=J(0, t)=A D \partial_{x} c(0, t)$, so after applying asymptotic matching:

$$
-\partial_{x} c(0, t)+4 s \tilde{N}(t) c(0, t)=0,
$$

where $\tilde{N}=N / A$ is the surface density of free receptors. Though equation (7) in principle only holds if the receptor surface density is sufficiently low, the boundary condition (8) was also found to describe the problem very accurately at very high receptor surface densities (Geurts and Wiegel, 1987). This boundary condition expresses the fact that not every encounter of a ligand with the sensor surface leads to binding of the ligand. As more and more complexes are formed the binding of additional ligands becomes increasingly difficult. Notice that equation (2) can thus be rewritten as:

$$
\frac{\mathrm{d} \tilde{n}(t)}{\mathrm{d} t}=4 s D\left(\tilde{N}_{0}-\tilde{n}(t)\right) c(0, t)-\gamma \tilde{n}(t),
$$

where $\tilde{N}_{0}=N_{0} / A$. Hence it may be inferred that the reaction's association constant is given by $4 s D$, as has been shown before for the circular disk case (Wiegel, 1983).

For convenience the variables are transformed to a dimensionless form. Let:

$$
T=\frac{D}{l^{2}} t ; \quad \xi=\frac{x}{l} ; u(\xi, T)=\frac{c(\xi, T)}{c_{0}} ; g(T)=\frac{\tilde{n}(T)}{\tilde{N}_{0}},
$$

and the model may be summarized as: 


$$
\begin{gathered}
\partial_{T} u(\xi, T)=\partial_{\xi \xi} u(\xi, T)+\tilde{\gamma} \frac{\tilde{N}_{0}}{l c_{0}} g(T) \delta(\xi-\tilde{\varepsilon}), \\
u(\xi, 0)=1, \\
u(1, T)=1(\text { Type } I) \text { or } \partial_{\xi} u(1, T)=0(\text { Type II), } \\
-\partial_{\xi} u(0, T)+\frac{1}{\alpha(T)} u(0, T)=0,
\end{gathered}
$$

where $\tilde{\gamma}=\gamma l^{2} / D, \tilde{\varepsilon}=\varepsilon / l$ and:

$$
\alpha(T)=\frac{1}{4 s l \tilde{N}(T)}=\frac{1}{4 s l\left(\tilde{N}_{0}-\tilde{n}(T)\right)} .
$$

The kinetic reaction equation for the receptor-ligand complexes now reads:

$$
\frac{\mathrm{d} g(T)}{\mathrm{d} T}=\frac{l c_{0}}{\tilde{N}_{0}} \partial_{\xi} u(0, T)-\tilde{\gamma} g(T) .
$$

Our main interest will be with the surface coverage $g(T)$, and it is clear from the above that in order to determine this surface coverage we need to solve the partial differential equation (11), and to integrate equation (16) using this solution. The case in which the receptor-ligand reaction is irreversible, i.e. $\gamma=0$, was treated separately and published elsewhere (Geurts and Wiegel, 1988).

In the next section the stationary state ligand distribution and surface coverage are determined. The full analytical solution to the problem posed by equations (11)-(16) will be derived in Section 4 using the stationary state results.

3. Stationary State Surface Coverage. In this section the stationary state surface coverage is derived, beginning with type I sensors. The ligand distribution in the stationary state $u_{s}(\xi)$ satisfies:

$$
\partial_{\xi \xi} u_{s}(\xi)+\tilde{\gamma} \frac{\tilde{N}_{0}}{l c_{0}} g(\infty) \delta(\xi-\tilde{\varepsilon})=0,
$$

where $g(\infty)$ denotes the limiting value of $g(T)$ as $T \rightarrow \infty$. For type I sensors the boundary condition at $\xi=1$ is:

$$
u_{s}(1)=1 \text {. }
$$


Also, the condition at $\xi=0$ can be written as:

$$
-\partial_{\xi} u_{s}(0)+\frac{1}{\alpha(0)} u_{s}(0)=\frac{g(\infty)}{\alpha(0)} u_{s}(0),
$$

where use was made of equations (14) and (15). The stationary state surface coverage follows from equation (16) as:

$$
g(\infty)=\frac{l c_{0}}{\tilde{\gamma} \tilde{N}_{0}} \partial_{\xi} u_{s}(0) .
$$

It is straightforward to integrate equation (17) subject to equations (18) and (19). One finds:

$$
\begin{array}{rlrl}
u_{s}(\xi) & =\partial_{\xi} u_{s}(0)\left[\xi+\frac{\alpha(0)}{1-g(\infty)}\right] ; & 0<\xi<\tilde{\varepsilon} \\
& =1 \quad ; \quad \tilde{\varepsilon}<\xi<1 .
\end{array}
$$

Since the ligand distribution is continuous one finds from matching equations (21) and (22) at $\xi=\tilde{\varepsilon}$ :

$$
\partial_{\xi} u_{s}(0)=\frac{1-g(\infty)}{\tilde{\varepsilon}(1-g(\infty))+\alpha(0)},
$$

and so, using equation (20) and taking the limit $\tilde{\varepsilon} \rightarrow 0$ one finds, after some elementary calculation:

$$
g(\infty)=\frac{y}{1+y},
$$

where:

$$
y=\frac{l c_{0}}{\alpha(0) \tilde{\gamma} \tilde{N}_{0}}=\frac{4 s D}{\gamma} c_{0} \equiv K c_{0},
$$

in which $K$ is the equilibrium constant for the receptor-ligand reaction. This is a standard equilibrium result which may be found elsewhere in the literature (see e.g. Eddowes, 1987). From a measurement of the stationary state surface coverage one obtains the parameter $y$ which is a combination of our model parameters $\tilde{\gamma}, \alpha(0)$ and $l c_{0} / \tilde{N}_{0}$. At a known concentration $c_{0}$ the ratio between the association and reverse reaction constants can be obtained.

Type II sensors can be treated in the same way. One has to replace equation (18) by:

$$
\partial_{\xi} u_{s}(1)=0 \text {, }
$$


since the ligand current at $\xi=1$ vanishes. One readily obtains:

$$
\begin{array}{rlrl}
u_{s}(\xi) & =\partial_{\xi} u_{s}(0)\left[\xi+\frac{\alpha(0)}{1-g(\infty)}\right] ; & 0<\xi<\tilde{\varepsilon} \\
& =B \quad ; \quad \tilde{\varepsilon}<\xi<1 .
\end{array}
$$

where $B$ is a constant to which a definite value is momentarily assigned. Using the condition that $u_{s}(\xi)$ must be continuous everywhere one finds, analogous to equation (23):

$$
\partial_{\xi} u_{s}(0)=B \frac{1-g(\infty)}{\tilde{\varepsilon}(1-g(\infty))+\alpha(0)} .
$$

The value for $B$ can be determined as follows. Since the system is closed [viz. equation (26)] the total number of ligands in the stationary state equals the number of ligands initially inserted in the medium. Initially, $A l c_{0}$ ligands are introduced into the system. As $T \rightarrow \infty$ part of these ligands is on the average bound to the sensor surface and the rest is free in the medium. This leads to the condition that:

$$
A l c_{0}=A \tilde{n}(\infty)+A l c_{0} \int_{0}^{1} u_{s}(\xi) \mathrm{d} \xi
$$

or:

$$
\int_{0}^{1} u_{s}(\xi) \mathrm{d} \xi+\frac{1}{\tilde{\gamma}} \partial_{\xi} u_{s}(0)=1
$$

After some calculation one finds, in the limit $\tilde{\varepsilon} \rightarrow 0$ :

$$
B=\frac{\alpha(0) \tilde{\gamma}}{(1-g(\infty))+\alpha(0) \tilde{\gamma}},
$$

and hence, through equations (20), (29) and (32), one obtains a quadratic equation for the stationary state surface coverage:

$$
g^{2}(\infty)-g(\infty)\left[1+\alpha(0) \tilde{\gamma}+\frac{l c_{0}}{\tilde{N}_{0}}\right]+\frac{l c_{0}}{\tilde{N}_{0}}=0
$$

The relevant root following from equation (33) is:

$$
g(\infty)=\frac{1}{2}\left[\left(1+\alpha(0) \tilde{\gamma}+\frac{l c_{0}}{\tilde{N}_{0}}\right)-\left\{\left(1+\alpha(0) \tilde{\gamma}+\frac{l c_{0}}{\tilde{N}_{0}}\right)^{2}-4 \frac{l c_{0}}{\tilde{N}_{0}}\right\}^{1 / 2}\right]
$$


Notice that $g(\infty)$ is determined by two parameters; the ratio between the number of ligands in the system and the total number of receptors $l c_{0} / \tilde{N}_{0}$ and $\alpha(0) \tilde{\gamma}$. Recalling the definitions of $\tilde{\gamma}$ and $\alpha(0)$ one notices that $\alpha(0) \tilde{\gamma}=$ $(\gamma / 4 s D)\left(l / \tilde{N}_{0}\right)=(1 / K)\left(l / \tilde{N}_{0}\right)$. So $\alpha(0) \tilde{\gamma}$ is the ratio between the reverse reaction and association constants multiplied by the ratio between the thickness $(l)$ of the fluid layer containing the ligands and the receptor density $\tilde{N}_{0}$.

In Fig. $1 g(\infty)$ is plotted as a function of $\alpha(0) \tilde{\gamma}$ at various values of $l c_{0} / \tilde{N}_{0}$. If $\alpha(0) \tilde{\gamma}$ increases, the surface coverage decreases, as one may expect. As the

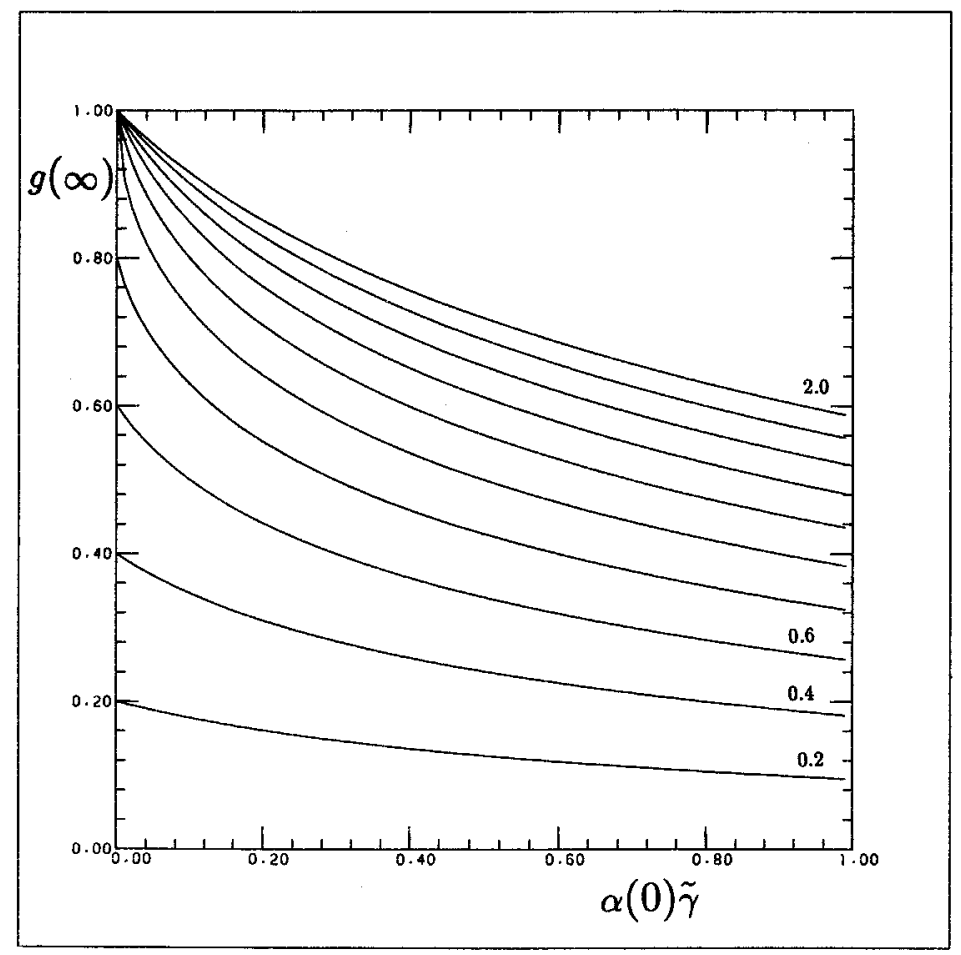

Figure 1. The stationary state surface coverage for type II sensors $g(\infty)$ as a function of $\alpha(0) \tilde{\gamma}$ for various values of the ligand-receptor ratio $l c_{0} / \tilde{N}_{0}$. The values used for this ratio are indicated near the corresponding lines.

reverse reaction rate $\gamma$ increases complexes are more likely to disintegrate. Likewise, an increase in $\alpha(0)$ corresponds (roughly speaking) to a decrease in $\tilde{N}_{0}$ and the binding of a ligand becomes less likely. Hence, the decrease in $g(\infty)$. Moreover, the maximal surface coverage is obtained as $\alpha(0) \tilde{\gamma}=0$, and is given by the minimum of $l c_{0} / \widetilde{N}_{0}$ and 1 , which implies full coverage only as $l c_{0} / \tilde{N}_{0} \geq 1$. From measurements at known ligand concentration, the parameters $l / \tilde{N}_{0}$ and the ratio between the reverse reaction and association constants can be obtained. Let $Z_{1}$ be the stationary state surface coverage obtained for a sample 
with concentration $c_{0}^{(1)}$ and $Z_{2}$ for a sample with concentration $c_{0}^{(2)}$, then one finds after some calculation, using equation (34):

$$
\alpha(0) \tilde{\gamma}=\frac{c_{0}^{(2)} Z_{1}-c_{0}^{(1)} Z_{2}}{c_{0}^{(1)} \frac{Z_{2}}{1-Z_{2}}-c_{0}^{(2)} \frac{Z_{1}}{1-Z_{1}}},
$$

and:

$$
\frac{l}{\tilde{N}_{0}}=\frac{\left(1-Z_{1}\right)-\left(1-Z_{2}\right)}{c_{0}^{(1)} \frac{1-Z_{1}}{Z_{1}}-c_{0}^{(2)} \frac{1-Z_{2}}{Z_{2}}} .
$$

Dividing equation (35) by (36) gives $\gamma / 4 s D$.

In the next section the analytical solution for the full problem posed by equations (11)-(16) is derived using the stationary state solution derived above.

4. Analytical Solution for the Time-dependent Surface Coverage. In order to obtain the analytical solution to the problem posed by equations (11)-(16) one must perform a lengthy and tedious calculation. We will first discuss this calculation in detail and then determine the short time behaviour explicitly. Let:

$$
u(\xi, T)=u_{s}(\xi)+U(\xi, T),
$$

then one may easily verify that $U(\xi, T)$ satisfies:

$$
\begin{gathered}
\partial_{T} U(\xi, T)=\partial_{\xi \xi} U(\xi, T)+\tilde{\gamma} \frac{\tilde{N}_{0}}{l c_{0}}\{g(T)-g(\infty)\} \delta(\xi-\tilde{\varepsilon}) \\
U(\xi, 0)=1-u_{s}(\xi) \\
U(1, T)=0(\text { Type } I) \text { or } \partial_{\xi} U(1, T)=0(\text { Type II)} \\
-\partial_{\xi} U(0, T)+\frac{1}{\alpha(0)} U(0, T)=\frac{1}{\alpha(0)}\{g(T)-g(\infty)\} u_{s}(0)+\frac{1}{\alpha(0)} g(T) U(0, T)
\end{gathered}
$$

The above problem [equations (38)-(41)] can be solved by reducing it to three simpler problems (Dettman, 1962). Put:

$$
U(\xi, T)=v(\xi, T)+w(\xi, T)+z(\xi, T),
$$


where $v(\xi, T)$ satisfies:

$$
\begin{gathered}
\partial_{T} v(\xi, T)=\partial_{\xi \xi} v(\xi, T), \\
v(\xi, 0)=1-u_{s}(\xi), \\
v(1, T)=0(\text { Type } I) \text { or } \partial_{\xi} v(1, T)=0(\text { Type II }), \\
-\partial_{\xi} v(0, T)+\frac{1}{\alpha(0)} v(0, T)=0,
\end{gathered}
$$

and $w(\xi, T)$ is defined by:

$$
\begin{gathered}
\partial_{T} w(\xi, T)=\partial_{\xi \xi} w(\xi, T)+\tilde{\gamma} \frac{\tilde{N}_{0}}{l c_{0}}\{g(T)-g(\infty)\} \delta(\xi-\tilde{\varepsilon}), \\
w(\xi, 0)=0 \\
w(1, T)=0\left(\text { Type I) or } \partial_{\xi} w(1, T)=0(\text { Type II })\right. \\
-\partial_{\xi} w(0, T)+\frac{1}{\alpha(0)} w(0, T)=0 .
\end{gathered}
$$

Finally the function $z(\xi, T)$ obeys:

$$
\begin{gathered}
\partial_{T} z(\xi, T)=\partial_{\xi \xi} z(\xi, T), \\
z(\xi, 0)=0, \\
z(1, T)=0(\text { Type } I) \text { or } \partial_{\xi} z(1, T)=0(\text { Type } I), \\
-\partial_{\xi} z(0, T)+\frac{1}{\alpha(0)} z(0, T)=\frac{1}{\alpha(0)}\{g(T)-g(\infty)\} u_{s}(0) \\
+\frac{1}{\alpha(0)} g(T)\{v(0, T)+w(0, T)+z(0, T)\} .
\end{gathered}
$$

Clearly $U(\xi, T)$, as defined by equation (42), satisfies the problem posed by equations (38)-(41). Hence, if $v, w$ and $z$ can be found, the analytical solution for the ligand distribution will be obtained, and hence, through an integration of equation (16) also the surface coverage $g(T)$. 
The calculation of the solutions $v, w$ and $z$ is quite lengthy, and we refer the reader to the appendix for details. For type I sensors one obtains:

$$
\begin{gathered}
v(\xi, T)=2 \tilde{\gamma} \frac{\tilde{N}_{0}}{l c_{0}} g(\infty) \sum_{j=1}^{\infty} \frac{\lambda_{j}(\tilde{\varepsilon}+\alpha(0))-g_{j}(\tilde{\varepsilon})}{\lambda_{j}^{2}\left(1+\alpha(0)+\alpha^{2}(0) \lambda_{j}^{2}\right)} g_{j}(\xi) e^{-\lambda_{j}^{2} T}, \\
w(\xi, T)=2 \tilde{\gamma} \frac{\tilde{N}_{0}}{l c_{0}} \sum_{j=1}^{\infty} \frac{g_{j}(\tilde{\varepsilon}) g_{j}(\xi)}{1+\alpha(0)+\alpha^{2}(0) \lambda_{j}^{2}} \int_{0}^{T} \mathrm{e}^{-\lambda_{j}^{2}(T-\mu)}\{g(\mu)-g(\infty)\} \mathrm{d} \mu \\
z(\xi, T)=\frac{2}{\alpha(0)} \sum_{j=1}^{\infty} \frac{g_{j}(0) g_{j}(\xi)}{1+\alpha(0)+\alpha^{2}(0) \lambda_{j}^{2}} \int_{0}^{T} \mathrm{e}^{-\lambda_{j}^{2}(T-\mu)}\left[\{g(\mu)-g(\infty)\} u_{s}(0)\right. \\
+g(\mu)\{v(0, \mu)+w(0, \mu)+z(0, \mu\}] \mathrm{d} \mu .
\end{gathered}
$$

where the eigenvalues $\left\{\lambda_{j}\right\}$ are the positive roots of:

$$
\tan \left(\lambda_{j}\right)=-\alpha(0) \lambda_{j}
$$

and the eigenfunctions $\left\{g_{j}\right\}$ are given by:

$$
g_{j}(\xi)=\sin \left(\lambda_{j} \xi\right)+\alpha(0) \lambda_{j} \cos \left(\lambda_{j} \xi\right) .
$$

The solution given by equations (55)-(57) is somewhat complicated and implicit. The functions $w$ and $z$ are determined in terms of their corresponding values at the sensor surface, integrated over all history and the surface coverage $g(T)$. Putting $\xi=0$ in equations (56) and (57), a consistency relation for $w(0, T)$ and $z(0, T)$ in terms of $g(T)$ is obtained, taking the form of a system of coupled non-linear integral equations. If $g(T)$ is now expressed in terms of this solution at the sensor surface as well, this set of equations is closed.

Integrating equation (16) and using equation (37) one has:

$$
g(T)=\frac{l c_{0}}{\tilde{N}_{0}} \int_{0}^{T} \mathrm{e}^{-\bar{\gamma}(T-\mu)}\left[\partial_{\xi} u_{s}(0)+\partial_{\xi} U(0, \mu)\right] \mathrm{d} \mu .
$$

Using equation (41) this can be rewritten as:

$$
\begin{aligned}
g(T)= & \frac{l c_{0}}{\tilde{\gamma} \tilde{N}_{0}} \partial_{\xi} u_{s}(0)\left[1-\mathrm{e}^{-\bar{\gamma} T}\right] \\
& +\frac{l c_{0}}{\alpha(0) \tilde{N}_{0}} \int_{0}^{T} \mathrm{e}^{-\bar{\gamma}(T-\mu)}\left[(1-g(\mu)) U(0, \mu)-(g(\mu)-g(\infty)) u_{s}(0)\right] .
\end{aligned}
$$

Hence, $w(0, T), z(0, T)$ and $g(T)$ are known implicitly and obey the system of non-linear integral equations (56), (57) and (61) upon setting $\xi=0$. A numerical 
approach to this system of equations will be formulated in Section 5. The short time behaviour of $g(T)$ can be calculated explicitly using equation (61).

Since $g(0)=0$ one has the first order Taylor expansion:

$$
g(T)=g^{\prime}(0) T+\mathcal{O}\left(T^{2}\right) .
$$

Moreover, differentiating equation (61) with respect to $T$, and, setting $T=0$, one finds:

$$
\begin{aligned}
g^{\prime}(0) & =\frac{l c_{0}}{\tilde{N}_{0}} \partial_{\xi} u_{s}(0)+\frac{l c_{0}}{\alpha(0) \tilde{N}_{0}}\left[U(0,0)+g(\infty) u_{s}(0)\right] \\
& =\frac{l c_{0}}{\alpha(0) \tilde{N}_{0}},
\end{aligned}
$$

where use was made of equations (19) and (39). This result is independent of the type of sensor used, and it shows that $g^{\prime}(0)$ is directly proportional to the (unknown) ligand concentration $c_{0}$ of the sample. Thus it is possible to determine this concentration $c_{0}$ from a measurement of $g(T)$ for $T \ll 1$, which makes the measurement of $c_{0}$ much faster than from a measurement of $g(\infty)$ as is usually done. This becomes more and more important as $c_{0}$ decreases since the stationary state sets in at a later time in that case.

The interpretation of equation (62) becomes more transparent if one writes it in terms of the original variables. One has:

$$
g(t)=4 s D c_{0} t+\mathcal{O}\left(\left(\frac{D t}{l^{2}}\right)^{2}\right)
$$

which shows that $g^{\prime}(0)$ is given by the reaction's association constant multiplied by the initial ligand concentration.

Type II sensors can be treated in exactly the same way. The expressions for $w$ and $z$ remain unaltered except that the eigenvalues $\left\{\lambda_{j}\right\}$ are now the positive roots of:

$$
\tan \left(\lambda_{j}\right)=\frac{1}{\alpha(0) \lambda_{j}}
$$

Since the stationary state $u_{s}(\xi)$ for type II sensors differs from that of type I sensors, the function $v$ is also changed slightly and is given by:

$$
v(\xi, T)=2\left(1-u_{s}(0)\right) \sum_{j=1}^{\infty} \frac{g_{j}(\xi)}{\lambda_{j}\left(1+\alpha(0)+\alpha^{2}(0) \lambda_{j}\right)} \mathrm{e}^{-\lambda_{j}^{2} T},
$$

in the limit $\tilde{\varepsilon} \rightarrow 0$. Notice that for type II sensors $v \neq 0$ as was the case with type I sensor as $\tilde{\varepsilon} \rightarrow 0$. 
In the next section, the system of non-linear integral equations determining $w(0, T), z(0, T)$ and $g(T)$ is described and a numerical approach from which these functions follow is proposed.

5. A System of Non-Linear Integral Equations Determining the Surface Coverage. In this section we consider the system of integral equations determining $w(0, T), z(0, T)$ and $g(T)$ in the limit in which $\tilde{\varepsilon} \rightarrow 0$. A convergent algorithm for this problem is formulated and its implementation described.

As was shown in the previous section, the surface coverage $g(T)$ is determined by a system of coupled non-linear integral equations which takes the following form as $\tilde{\varepsilon} \rightarrow 0$ :

$$
\begin{aligned}
w(0, T)= & 2 \alpha^{2}(0) \tilde{\gamma} \frac{\tilde{N}_{0}}{l c_{0}} \sum_{j=1}^{\infty} \frac{\lambda_{j}^{2}}{1+\alpha(0)+\alpha^{2}(0) \lambda_{j}^{2}} \int_{0}^{T} \mathrm{e}^{-\lambda_{j}^{2}(T-\mu)}\{g(\mu)-g(\infty)\} \mathrm{d} \mu, \\
z(0, T)= & 2 \alpha(0) \sum_{j=1}^{\infty} \frac{\lambda_{j}^{2}}{1+\alpha(0)+\alpha^{2}(0) \lambda_{j}^{2}} \int_{0}^{T} \mathrm{e}^{-\lambda_{j}^{2}(T-\mu)}\left\{(g(\mu)-g(\infty)) u_{s}(0)\right. \\
& +g(\mu)[v(0, \mu)+w(0, \mu)+z(0, \mu)]\} \mathrm{d} \mu, \\
g(T)= & \frac{l c_{0}}{\tilde{\gamma} \tilde{N}_{0}} \partial_{\xi} u_{s}(0)\left[1-\mathrm{e}^{-\bar{\gamma} T}\right]+\frac{l c_{0}}{\alpha(0) \tilde{N}_{0}} \int_{0}^{T} \mathrm{e}^{-\bar{\gamma}(T-\mu)} \\
& \left.\times\left\{(1-g(\mu))[v(0, \mu)+w(0, \mu)+z(0, \mu)]-(g(\mu)-g(\infty)) u_{s}(0)\right)\right\} \mathrm{d} \mu .(6 g)
\end{aligned}
$$

It does not seem possible to derive $w(0, T), z(0, T)$ and $g(T)$ analytically from equations (67)-(69). However, by iteration it is possible to determine these functions numerically.

Symbolically, one may write equations (67)-(69) as:

$$
\begin{gathered}
w(0, T)=I_{1}(g(T)), \\
z(0, T)=I_{2}(g(T), w(0, T), z(0, T) ; v(0, T)), \\
g(T)=\frac{l c_{0}}{\tilde{\gamma} \tilde{N}_{0}} \partial_{\xi} u_{s}(0)\left[1-\mathrm{e}^{-\bar{\gamma} T}\right]+I_{3}(g(T), w(0, T), z(0, T) ; v(0, T)),
\end{gathered}
$$

where $I_{1}, I_{2}$ and $I_{3}$ are operators defined through equations (67), (68) and (69) respectively. A sequence of functions $\left(w_{k}(0, T), z_{k}(0, T), g_{k}(T)\right)$ which converges in the maximum norm to the desired solution can be defined as follows:

$$
w_{k}(0, T)=I_{1}\left(g_{k-1}(T)\right),
$$




$$
z_{k}(0, T)=I_{2}\left(g_{k-1}(T), w_{k-1}(0, T), z_{k-1}(0, T) ; v(0, T)\right),
$$

for $k=1,2, \ldots$ with the initialization $w_{0}(0, T)=z_{0}(0, T)=0$ and:

$$
g_{k}(T)=\frac{l c_{0}}{\tilde{\gamma} \tilde{N}_{0}} \partial_{\xi} u_{s}(0)\left[1-\mathrm{e}^{-\bar{\gamma} T}\right]+I_{3}\left(g_{k-1}(T), w_{k-1}(0, T), z_{k-1}(0, T) ; v(0, T)\right),
$$

for $k=2,3, \ldots$ and:

$$
g_{0}(T)=g_{1}(T)=\frac{l c_{0}}{\tilde{\gamma} \tilde{N}_{0}} \partial_{\xi} u_{s}(0)\left[1-\mathrm{e}^{-\tilde{\gamma} T}\right]
$$

Typically, in about 10 iterations this scheme converged to the desired solution with an error of $\mathcal{O}\left(10^{-3}\right)$. As the reader may anticipate, this calculation is quite time consuming. A significant reduction in the computer time needed can be obtained by considering the integrals in equations (67)-(69). These integrals are of the form:

$$
h_{j}(T)=\int_{0}^{t} \mathrm{e}^{-\lambda_{j}^{2}(T-\mu)} G(\mu) \mathrm{d} \mu,
$$

which is equivalent to:

$$
h_{j}^{\prime}(T)=G(T)-\lambda_{j}^{2} h_{j}(T) ; \quad h_{j}(0)=0 .
$$

This latter differential equation can be integrated quite quickly and reliably using Euler's algorithm, i.e. we put:

$$
h_{j}(T+\Delta T)=h_{j}(T)+h_{j}^{\prime}(T) \Delta T,
$$

provided the integration time step $\Delta T$ is small compared to $1 / \lambda_{j}^{2}$. Typically $\Delta T \approx \mathcal{O}\left(1 /\left(5 \lambda_{j}^{2}\right)\right)$ was found to be appropriate.

In the next section results obtained for the time-dependent surface coverage are discussed.

6. Discussion of Results. Above, the surface coverage $g(T)$ was related explicitly to the initial ligand concentration $c_{0}$ and reaction and sensor parameters. As stated in the introduction this surface coverage is the measurable quantity in an experimental situation. Hence, through the relation obtained between $g(T)$ and $c_{0}$ it is possible to measure (indirectly) this (unknown) ligand concentration. Also, these predictions for the surface coverage can be tested experimentally. First results for $g(T)$ for type I sensors are presented, followed by type II sensors. 


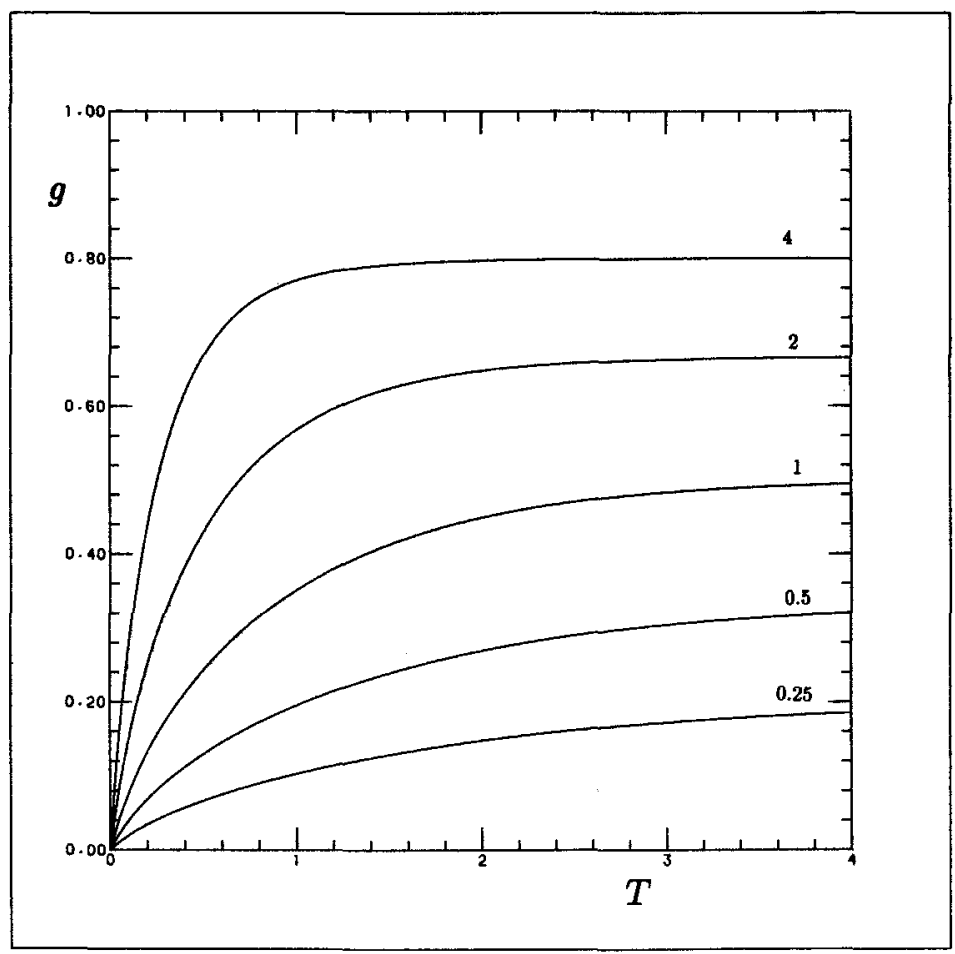

Figure 2. The surface coverage $g$ as a function of time $T$ for type I sensors showing the dependence on variations of the ligand-receptor ratio $l c_{0} / \tilde{N}_{0}$. In these calculations we kept $\alpha(0)=\tilde{\gamma}=1$ and indicated the values for the ligand-receptor ratio used near the corresponding lines. These results, as well as those presented in Figs $3-7$, were obtained using the iteration scheme as defined by equations (73)-(76).

In Fig. 2 the time-dependent surface coverage is plotted in the case in which the ligand-receptor ratio $l c_{0} / \tilde{N}_{0}$ was varied for type I sensors. Notice that, consistent with equation (24), the stationary value increases as $l c_{0} / \tilde{N}_{0}$ increases, as does the derivative $g^{\prime}(0)$, consistent with equation (63). Further notice that the stationary state sets in at a later time if $l c_{0} / \tilde{N}_{0}$ decreases. Typically the stationary state sets in as $T=\mathcal{O}(1-10)$. Since $D=\mathcal{O}\left(10^{-10}\right) \mathrm{m}^{2} \mathrm{~s}^{-1}$ (Eddowes, 1987; Wiegel, 1983) and the fluid layer typically is $l=\mathscr{O}\left(10^{-3}\right)-\mathcal{O}\left(10^{-4}\right) \mathrm{m}$ (Eddowes, 1987) this implies that it takes several hours before the stationary state sets in. This makes an experimental determination of the ligand concentration from this type of measurements rather tedious, especially if the concentration is low. In Fig. 3 results are shown for type I sensors for the case in which $\alpha(0)$ was varied. Roughly speaking an increase in $\alpha(0)$ corresponds to experimental situations in which the receptor density $\tilde{N}_{0}$ decreases. Hence, the decrease in $g(T)$ as $\alpha(0)$ is increased, since in these situations it becomes less likely for a ligand to be bound. Finally, in Fig. $4 g(T)$ is plotted showing the 


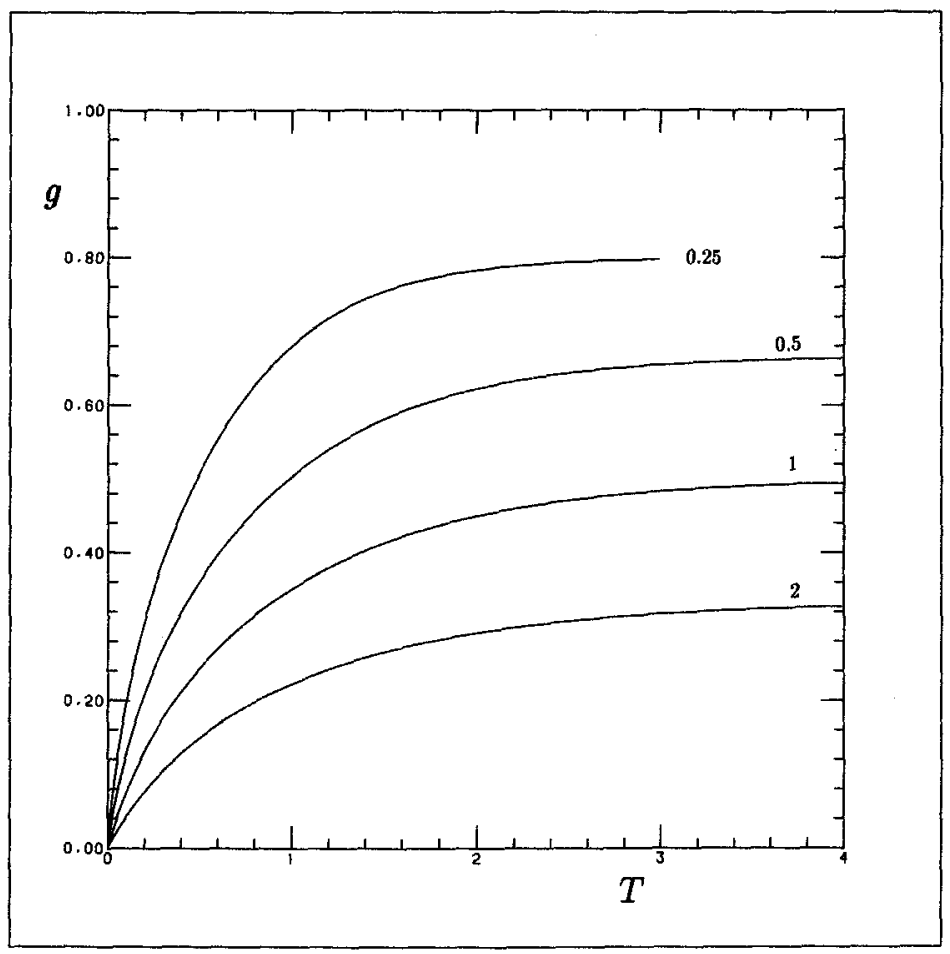

Figure 3. The surface coverage $g$ as a function of time $T$ for type I sensors showing the dependence of variations in $\alpha(0)$. We used $l c_{0} / \tilde{N}_{0}=\tilde{\gamma}=1$ and the corresponding values used for $\alpha(0)$ are indicated near the corresponding lines.

influence of varying $\tilde{\gamma}$. Notice that, consistent with equations (63) and (64) the short time behaviour is independent of these variations. Also, $g(T)$ decreases as $\tilde{\gamma}$ is increased as one may expect. Finally, the stationary state sets in at a later time if $\tilde{\gamma}$ is decreased.

Turning to the results obtained for type II sensors, the behaviour of $g(T)$ is qualitatively the same as for type I sensors though $g(T)$ turns out to be somewhat lower as compared to the corresponding behaviour for type I sensors. Figure 5 shows the influence of varying $l c_{0} / \tilde{N}_{0} ;$ Fig. 6 exemplifies the surface coverage as $\alpha(0)$ is varied, and finally in Fig. $7 \tilde{\gamma}$ is varied. In most experimental situations one deals with type II sensors. The results obtained differ significantly from those predicted by Eddowes (Eddowes, 1987). An experimental test of these predictions could be used to fix the model parameters used.

The author is greatly indebted to Dr B.S. F. Altenburg of the Department of Applied Physics (TN/BFT) of Twente University for several stimulating discussions and explanation of the basic biochemical concepts involved in the operation of plasmonsensors. 


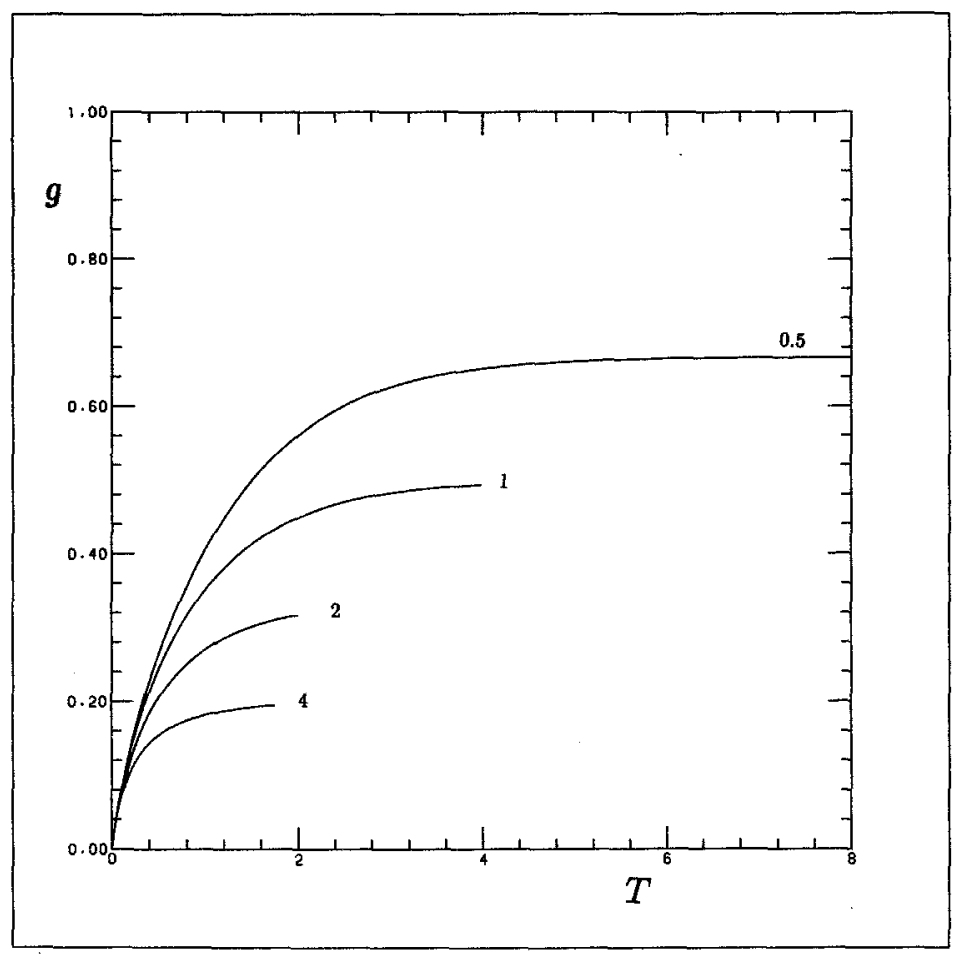

Figure 4. The surface coverage $g$ as a function of time $T$ for type I sensors for the case in which $\tilde{\gamma}$ was varied keeping $\alpha(0)=l c_{0} / \widetilde{N}_{0}=1$. The values used for $\tilde{\gamma}$ are indicated near the corresponding lines.

\section{APPENDIX}

In this appendix the solutions $v, w$ and $z$ to the problems posed by equations $(43)-(46),(47)-(50)$ and (51)-(54) respectively are derived. The calculation is shown in detail for type I sensors and changes in this derivation for type II sensors are indicated afterwards.

We represent the function $v(\xi, T)$ as:

$$
v(\xi, T)=\sum_{j=1}^{\infty}\left[A_{j} \sin \left(\lambda_{j} \xi\right)+B_{j} \cos \left(\lambda_{j} \xi\right)\right] \mathrm{e}^{-\lambda_{j}^{2} T},
$$

with $\left\{\lambda_{j}\right\}$ positive real numbers. The boundary conditions [equations (45) and (46)] imply:

$$
\begin{gathered}
A_{j} \sin \left(\lambda_{j}\right)+B_{j} \cos \left(\lambda_{j}\right)=0, \\
-\lambda_{j} A_{j}+\frac{1}{\alpha(0)} B_{j}=0 .
\end{gathered}
$$

Hence non-trivial solutions are obtained only if:

$$
\tan \left(\lambda_{j}\right)=-\alpha(0) \lambda_{j}
$$




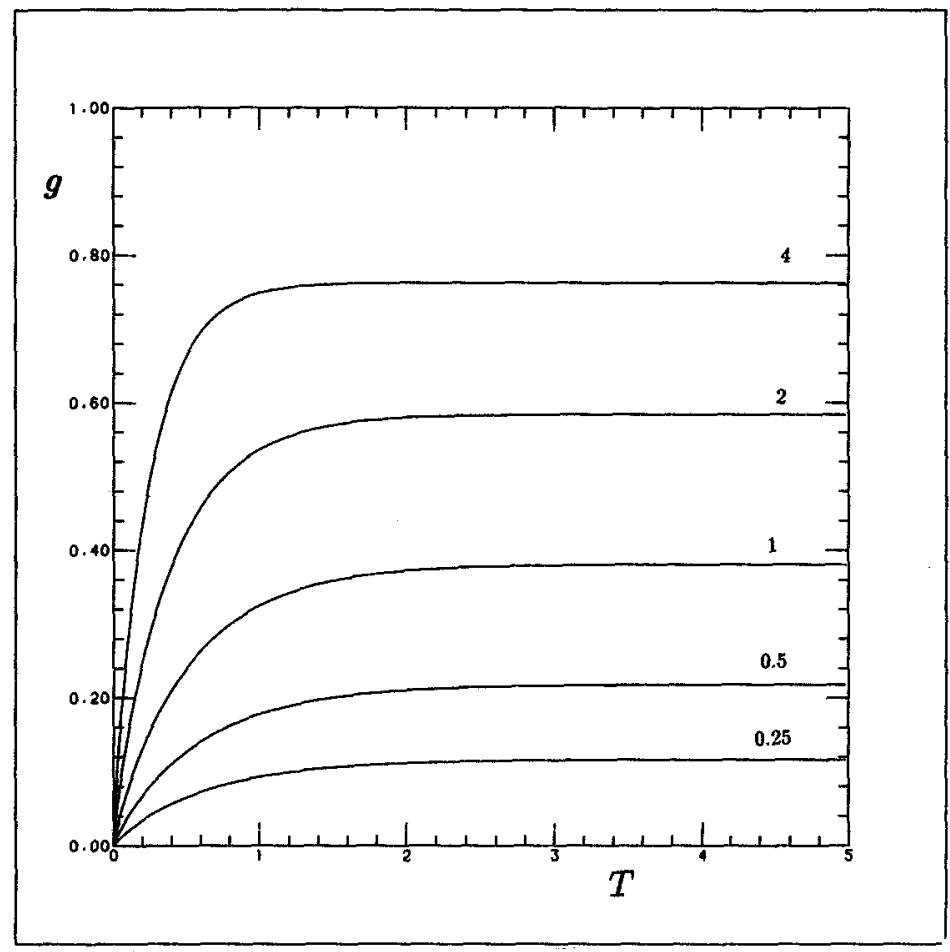

Figure 5. The surface coverage $g$ as a function of time $T$ for type II sensors. We varied $l c_{0} / \tilde{N}_{0}$ and kept $\alpha(0)=\tilde{\gamma}=1$. The values for $l c_{0} / \tilde{N}_{0}$ used are indicated near the corresponding lines.

and $v(\xi, T)$ can be further specified as:

$$
v(\xi, T)=\sum_{j=1}^{\infty} A_{j} g_{j}(\xi) \mathrm{e}^{-\lambda_{j}^{2} T},
$$

where:

$$
g_{j}(\xi)=\sin \left(\lambda_{j} \xi\right)+\alpha(0) \lambda_{j} \cos \left(\lambda_{j} \xi\right) .
$$

Using the orthogonality relation:

$$
\int_{0}^{1} g_{j}(\xi) g_{k}(\xi) \mathrm{d} \xi=\frac{1}{2}\left[1+\alpha(0)+\alpha^{2}(0) \lambda_{j}^{2}\right] \delta_{j, k},
$$

where $\delta_{j, k}$ is Kronecker's delta, we may derive the coefficients $\left\{A_{j}\right\}$ from:

$$
A_{j} \int_{0}^{1} g_{j}^{2}(\xi)=\frac{\tilde{\gamma} \tilde{N}_{0}}{l c_{0}} g(\infty) \int_{0}^{1} g_{j}(\xi)(\tilde{\varepsilon}-\xi) \mathrm{d} \xi,
$$

resulting in equation (55).

The derivation of $w(\xi, T)$ is somewhat more complicated, but can be performed using Duhamel's theorem which states that (Carslaw and Jaeger, 1959): 


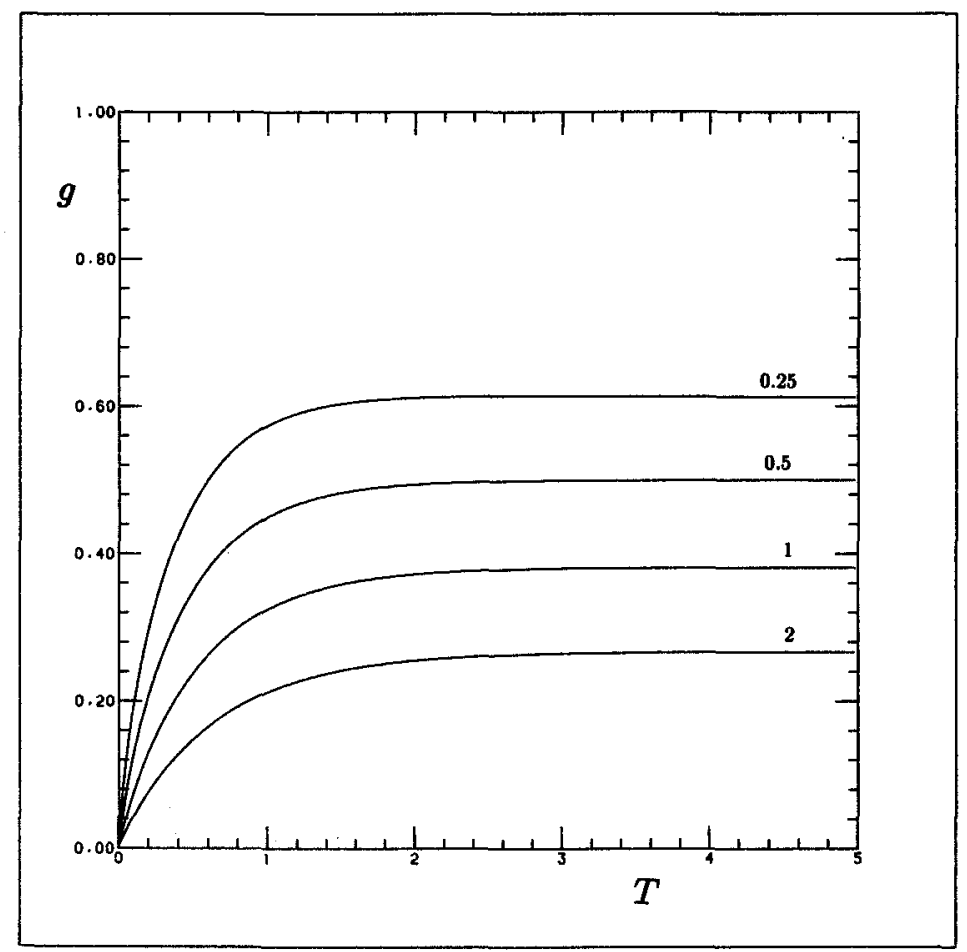

Figure 6. The surface coverage $g$ as a function of time $T$ for type II sensors. We varied $\alpha(0)$ and kept $l c_{0} / \bar{N}_{0}=\tilde{\gamma}=1$. The values for $\alpha(0)$ used are indicated near the corresponding lines.

$$
w(\xi, T)=\int_{0}^{T} \frac{\partial w^{*}}{\partial T}(\xi, T-\mu ; \mu) \mathrm{d} \mu
$$

in which $w^{*}(\xi, T ; \mu)$ satisfies:

$$
\partial_{T} w^{*}(\xi, T ; \mu)=\partial_{\xi \xi} w^{*}(\xi, T ; \mu)+F(\mu) \delta(\xi-\tilde{\varepsilon}),
$$

and initial and boundary conditions as in equations (48)-(50). In this $\mu>0$ has a fixed value and:

$$
F(\mu)=\tilde{\gamma} \frac{\tilde{N}_{0}}{l c_{0}}\{g(\mu)-g(\infty)\} .
$$

The stationary state solution $w_{s}^{*}(\xi ; \mu)$ can be derived straightforwardly and is given by:

$$
\begin{aligned}
w_{s}^{*}(\xi ; \mu) & =F(\mu) \frac{(1-\tilde{\varepsilon})(\xi+\alpha(0))}{1+\alpha(0)}: \quad 0<\xi<\tilde{\varepsilon} \\
& =F(\mu) \frac{(1-\xi)(\tilde{\varepsilon}+\alpha(0))}{1+\alpha(0)}: \quad \tilde{\varepsilon}<\xi<1 .
\end{aligned}
$$

Putting: 


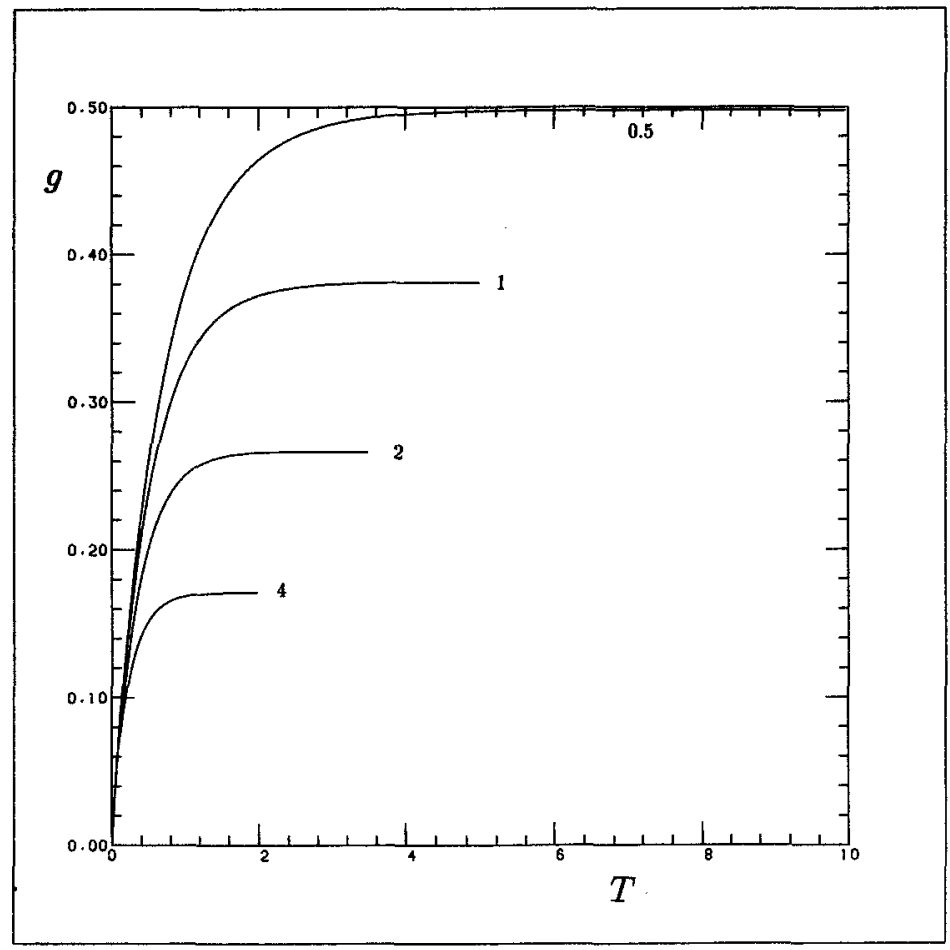

Figure 7. The surface coverage $g$ as a function of time $T$ for type II sensors. We varied $\tilde{\gamma}$ and kept $l c_{0} / \tilde{N}_{0}=\alpha(0)=1$. The values for $\tilde{\gamma}$ used are indicated near the corresponding lines.

$$
w^{*}(\xi, T ; \mu)=w_{s}^{*}(\xi ; \mu)+\sum_{j=1}^{\infty} A_{j}(\mu) g_{j}(\xi) \mathrm{e}^{-\lambda_{j}^{2} T}
$$

one can easily show that $w^{*}$ satisfies equation (A10) provided the eigenvalues satisfy equation (A4). After matching to the initial condition using equation (A7) one obtains:

$$
A_{j}(\mu)=-2 F(\mu) \frac{g_{j}(\tilde{E})}{\lambda_{j}^{2}\left(1+\alpha(0)+\alpha^{2}(0) \lambda_{j}^{2}\right)},
$$

and hence after applying equation (A9) we obtain equation (56).

Finally $z(\xi, T)$ can also be derived using Duhamel's theorem. The corresponding $z^{*}(\xi, T ; \mu)$ satisfies equations (51)-(53) and equation (54) is to be replaced by:

$$
-\partial_{\zeta} z^{*}(0, T ; \mu)+\frac{1}{\alpha(0)} z^{*}(0, T ; \mu)=\Phi(\mu),
$$

where:

$$
\Phi(\mu)=\frac{1}{\alpha(0)}\{g(\mu)-g(\infty)\} u_{s}(0)+\frac{1}{\alpha(0)} g(\mu)\{v(0, \mu)+w(0, \mu)+z(0, \mu)\}
$$


The stationary state solution $z_{s}^{*}(\xi ; \mu)$ is readily derived to be:

$$
z_{s}^{*}(\xi ; \mu)=\Phi(\mu) \frac{\alpha(0)}{\alpha(0)+1}(1-\xi),
$$

and putting $z(\xi, T ; \mu)$ in a form similar to equation (A14) one may show, after some calculation, that $\left\{A_{j}(\mu)\right\}$ are given by:

$$
A_{j}(\mu)=-2 \alpha(0) \Phi(\mu) \frac{1}{\lambda_{j}\left(1+\alpha(0)+\alpha^{2}(0) \lambda_{j}^{2}\right)},
$$

and hence the solution [equation (57)].

Type II sensors can be treated completely analogously. One finds as the eigenvalue equation:

$$
\tan \left(\lambda_{j}\right)=\frac{1}{\alpha(0) \lambda_{j}}
$$

and slight changes in $w_{s}^{*}$ and $z_{s}^{*}$ which however do not affect the form of the solution. The function $v$ can be derived in the same way as was shown before for type I sensors.

\section{LITERATURE}

Carslaw, H. S. and J. C. Jaeger. 1959. Conduction of Heat in Solids. Oxford: Clarendon Press. DeLisi, C. and F. W. Wiegel. 1981. Proc. Natnl. Acad. Sci. U.S.A. 78, 5569.

Dettman, J. W. 1962. Mathematical Methods in Physics and Engineering. New York: McGrawHill.

Eddowes, M. J. 1987. Biosensors 3, 1.

Geurts, B. J. and F. W. Wiegel. 1987. Biophys. Chem. 28, 7. and - 1988. Biophys. Chem. 31, 317.

Stenberg, M., L. Stibbert and H. Nygren. 1986. J. theor. Biol. 120, 129.

Stollar, B. O. 1981. Arthritis Rheum. 24, 1010.

Stryer, L. 1981. Biochemistry, p. 790. San Francisco: Freeman.

Wiegel, F. W. 1983. Phys. Rep. 95, 287.

, B. J. Geurts and B. Goldstein. 1987. J. Phys. A20, 5205.

Received 14 July 1988 Revised 22 November 1988 